\title{
Subcision in the Treatment of Acne Scar in Iraqi Patients
}

\author{
Hayder R. Al-Hammamy, Abd-Allah S. Mohammad, Ihsan A. Al-Turfy* \\ Department of Dermatology and Venereology, College of Medicine, University of Baghdad, Baghdad, Iraq \\ Email: ${ }^{*}$ dr ihssanalturfy@yahoo.com
}

Received 2 February 2015; accepted 11 June 2015; published 16 June 2015

Copyright (C) 2015 by authors and Scientific Research Publishing Inc.

This work is licensed under the Creative Commons Attribution International License (CC BY). http://creativecommons.org/licenses/by/4.0/

(c) (i) Open Access

\section{Abstract}

Background: Acne vulgaris is a very common skin disease among young people which might be associated with scarring that has a great impact on the emotional, psychological and social life of the patients as it will go with them for life. Subcision is a new technique for the treatment of acne scars. Objective: To assess objectively and subjectively the efficacy and safety of subcision in the treatment of depressed acne scars. Patients and Methods: This is an open-label therapeutic trial. A total of 16 patients were enrolled in this study. Twelve were males and 4 were females. Their ages ranged from 19 - 39 with a mean of $26.64 \pm 5.64$. The duration of scar varied between 6 months and 10 years with a mean of $4.14 \pm 2.54$ years. subcision was done by introducing a sterile, hypodermic, 18-gauge needle. The needle was held by a three $\mathrm{ml}$ syringe for better orientation of the sharp tip of the needle. It was kept horizontal to the skin surface with the bevel up, and was introduced in a high sub dermal plane. The needle was slowly advanced parallel to the skin surface. Initially, rapid, repetitive linear back-and-forth motion of the needle makes the skin free of the underlying scar. This procedure was repeated in all directions in a fan-like manner. Results: According to Modified Sharquie's scoring system for grading acne scars, $4(25 \%)$ patients had severe grade, $11(68.8 \%)$ patients had moderate grade and only $1(6.2 \%)$ patient had mild grade. Evaluation at 6 months after treatment revealed that $8(50 \%)$ patients had mild acne scar, $7(43.8 \%)$ had moderate acne scar and only $1(6.2 \%)$ patient still had severe grade. This change in the grades was statistically significant $(\mathrm{p}$-value $\mathbf{= 0 . 0 1 7 1 3 4 4 1 )}$. The average score before treatment was $13.13 \pm$ 2.363; it became $9.50 \pm 2.944$ after 6 months. By paired t-test comparison, the difference in the score was statistically highly significant $(p=0.000044)$. Regarding the photographic assessment, the difference in the visual analogue scale before and after treatment was statistically significant ( $p$-value $=0.043823$ ). All patients were satisfied regarding subcision as treatment for their acne scars with variable degrees. In general the reported side effects were transient and vanished within 3 - 7 days apart from firm bumps which resolved within 12 weeks in all patients. Conclusion: Subcision appears to be simple, safe, minimally invasive, well-tolerated, and effective surgical procedure that provides significant long-term betterment for depressed acne scars especially

\footnotetext{
*Corresponding author.
}

How to cite this paper: Al-Hammamy, H.R., Mohammad, A.-A.S. and Al-Turfy, I.A. (2015) Subcision in the Treatment of Acne Scar in Iraqi Patients. Journal of Cosmetics, Dermatological Sciences and Applications, 5, 125-133. 


\title{
for the rolling type.
}

\section{Keywords}

\author{
Subcision, Acne, Scar
}

\section{Introduction}

Acne vulgaris is the most common skin disorder encountered by dermatologists in practice; many patients with inflammatory acne suffer from significant scarring which is disfiguring and difficult to treat [1]. Scarring may occur early regardless of the severity of acne. Close inspection of acne skin under a bright light can reveal some scarring in up to $90 \%$ of patients who attend a dermatologist but significant (socially noticeable) scarring occurs in about $22 \%$ of sufferers [2]. All types of acne, from papules, pustules through nodulocystic disease can cause scarring and adequate treatment must be started early. Even with the excellent treatment options available, scarring may occur. Acne scars can be classified as ice pick, rolling, and boxcar scar. They can be atrophic or hypertrophic. Acne scars can also be classified as mild, moderate, or severe depending on certain criteria such as number, depth, color, and the area of the face involved. Scarring has profound psychological and social effects [3][5]. The ultimate goal of treating acne scar is for improvement and not necessarily for complete cure. For this purpose both medical treatment and a variety of surgical interventions have been employed, including scar excision, dermobrasion, lasers, and fillers.

Subcision (subcutaneous incisionless surgery) is a relatively new method to treat scars [6].

\section{What Is Subcision?}

Definition: The term subcision describes a unique form of incisionless local subcutaneous under mining. Its action depends on two phenomena. First, release the skin from its attachments to deeper tissues. Second, the controlled trauma initiates wound healing with consequent formation of connective tissue which augments the depressed site [7] [8].

Historical back ground: It has been used by Spangler in 1957, Gottlieb in 1977, Koranda in 1989, and Hambley and Carruthers in 1992. Since 1995, subcision has been established as an effective means of correcting depressed acne scars and wrinkles [7] [9].

Indications: Subcision is best to treat rolling acne scars with normal-appearing overlying skin and it lacks of a sharply delineated border [7]. It is contraindicated for areas of active cystic acne disease under the scar, or active infection, and in patients with bleeding diathesis or a tendency toward keloid formation and for deep icepick scars.

\section{Aim of the Study}

To assess objectively and subjectively the efficacy and side effects of subcision in the treatment of moderate to severe depressed acne scars.

\section{Patients and Methods}

This open-label therapeutic trial was carried out in the Department of Dermatology and Venereology, Baghdad Teaching Hospital in Baghdad from April 2011 to December 2012. This study was approved by the Ethical Committee of our hospital and written cosents were signed by all patients. A total of 16 patients enrolled in this study. Twelve were males and 4 were females. Their ages ranged from 19-39 with a mean of $26.64 \pm 5.64$. The duration of scar varied between 6 months and 10 years with a mean of $4.14 \pm 2.54$ year. Patients with rolling or depressed acne scars including mainly moderate and severe grades were included in the study. The exclusion criteria were: patients with deep ice pick scars, those with active disease under the scar or active infection and those with history of keloid scarring after trauma or surgery, patients with bleeding diathesis, anticoagulant medication, diabetes mellitus, ischemic heart disease, and pregnant women. All patients were evaluated by full history and physical examination. 
Procedure: Local anesthesia was done by infiltration of a solution of $2 \%$ lidocaine with 1:200,000 epinephrine into the superficial sub cutis. Anesthesia was sufficient to permit needle entry of $1 \mathrm{~cm}$ beyond the border of the scar areas. Then 5 - $10 \mathrm{ml}$ of normal saline were injected into each cheek to produce tumescence. A sterile, hypodermic, 18-gauge needle was used for subcision. The needle was held by a three $\mathrm{ml}$ syringe for better orientation of the sharp tip of the needle. It was kept horizontal to the skin surface with the bevel up, and was introduced in a high sub dermal plane. The needle was slowly advanced parallel to the skin surface. Initially rapid, repetitive linear back-and-forth motion of the needle in order to make the skin free of the underlying scar.this procedure was repeated in all directions in a fan-like manner. Larger scarred areas were treated through three entry sites to achieve triangulation of the treatment zone and to make thorough soft tissue release. The free hand acted as a guide to subcision and was used as needed to pinch, stretch, or stabilize the treatment site. Caution was taken to remain superficial to deep vessels and the proximal branches of facial nerve. The back-and-forth motion of the needle parallel to the skin surface resulted in audible rasping and popping as the underside of the dermis is released from its attachment to the sub cutis. A visible lift of the scar was noted at end-point of the procedure. Direct, manual pressure was applied immediately after a site was treated and dressed, and pressure was maintained for several minutes to obtain hemostasis.

Follow up: Patients were seen regularly at 2 weeks, 4 weeks, 8 weeks, 12 weeks and 6 months. At each visit, the response for treatment was assessed, the side effects were recorded, photographs were taken, and the scoring for grading scarring acne vulgaris was recorded. Subsequent sessions, if needed, were done 4 weeks apart and patients were followed up as above.

Evaluation: Patients were evaluated objectively and subjectively regarding their response to treatment by the following methods:

Acne Severity Score: A scoring system for acne severity was adopted based on Sharquie et al. method [1] [5]. The score depends on the followings:

1) The number of scars: lesion counting was done and if the number of scars in the cheek areas was:

3 or less, it scores 1.

$4-6$, it scores 2.

7 - 12, it scores 3

More than 12, it scores 4.

2) The area of the face involved: the area of the cheek occupied by scars was estimated and if it was:

$1 / 4$ or less, it scores 1.

More than $1 / 4$ to $1 / 2$, it scores 2 .

More than $1 / 2$ to $3 / 4$, it scores 3 .

More than $3 / 4$, it scores 4 .

3) The type of the scar: the predominant type of acne scars was considered and scored as the following:

Flat scars, it scores 1.

Depressed scars, it scores 2.

Hypertrophic scars, it scores 3.

Keloid scars, it scores 4.

4) The color of the scars: the predominant color was considered and if it was:

Skin colored, it scores 1 .

Hypopigmented or erythematous, it scores 2.

Hyperpigmented, it scores 3.

Discolored (i.e. blue or gray), it scores 4.

5) The effect of scarring on the psyche: the impact of scarring on patients psyche was assessed and scored as the following:

Only mild discomfort, it scores 1.

Mild bothersome, it scores 2 .

Moderate bothersome, it scores 3.

Severe bothersome, it scores 4 .

Then the total score was counted by adding the five parameters for each patient.

The severity of acne scarring was graded as the following:

Mild if the total score is 5 - 9 .

Moderate if the total score is $10-14$.

Severe if the total score is 15 - 20. This score was calculated before treatment and at each visit. 
Photographic Assessment: Frontal, right and left views were taken using Sony-Digital Still Camera-W350, The photographs were assessed at the end of the study in a computer view by two independent, out of the study dermatologists, and they notified their opinions about the degree of improvement using visual analogue scale from 0 - 10.

Patient's Satisfaction: Each patient was asked to rate his/her satisfaction with the procedure at the end of the follow up period.

Satisfaction was graded from 0 - 4 as follows:

Grade 0: not satisfied.

Grade 1: partially satisfied.

Grade 2: moderately satisfied.

Grade 3: greatly but not fully satisfied.

Grade 4: fully or completely satisfied.

Side effects were recorded at each visit and after follow up.

Statistical Analysis: Chi square test was used to evaluate the change in the total scores and individual para-meters from base line. (Paired t-test was used to evaluate the average score and compare the visual analogue scale before and after treatment).

\section{Results}

The number of sessions needed ranged from 1-3 sessions. Ten (62.5\%) patients needed only one session, 4 (25\%) patients needed 2 sessions and 2 (12.5\%) patients needed 3 sessions. Regarding the total score, the number of patients with score 5 - 9 (mild acne )increased from one patient to 8 patients on follow up, while the number of patients with severe acne ( score $=15-20$ ) dropped from 4 patients at the start to one patient on follow up (Table 1). The difference was statistically significant $(\mathrm{p}=0.017)$. Regarding the area involved, 10 patients had more than half of the cheek area involved at the beginning which was reduced to only 3 patients on follow up. This was statistically significant (Table 1). The type of scarring was depressed in all patients prior to treatment. Seven of these patients their scars were elevated and became flat at the end of follow up. At the start of treatment 8 patients had hyper pigmented scars while 6 had normal color. At follow up 4 had hyper pigmented and 10 had normal color $(p=0.311)$. Regarding the effect of psyche: Eleven patients considered their scars to be moderately bothering some, 3 patients considered the condition as severely bothering some, these numbers were reduced to 5 and zero respectively on follow up $(p=0.0079)$ (Table 1$)$. Evaluation of photos was done by means of visual analogue scale (VAS). For all photos the scale decreased from $7.11+/-0.65$ to $4.45+/-1.6$ (p $=0.044$ ) (see Figures 1-5). None of our patients was fully satisfied or completely unsatisfied with the treatment. Fifty percent were moderately satisfied.

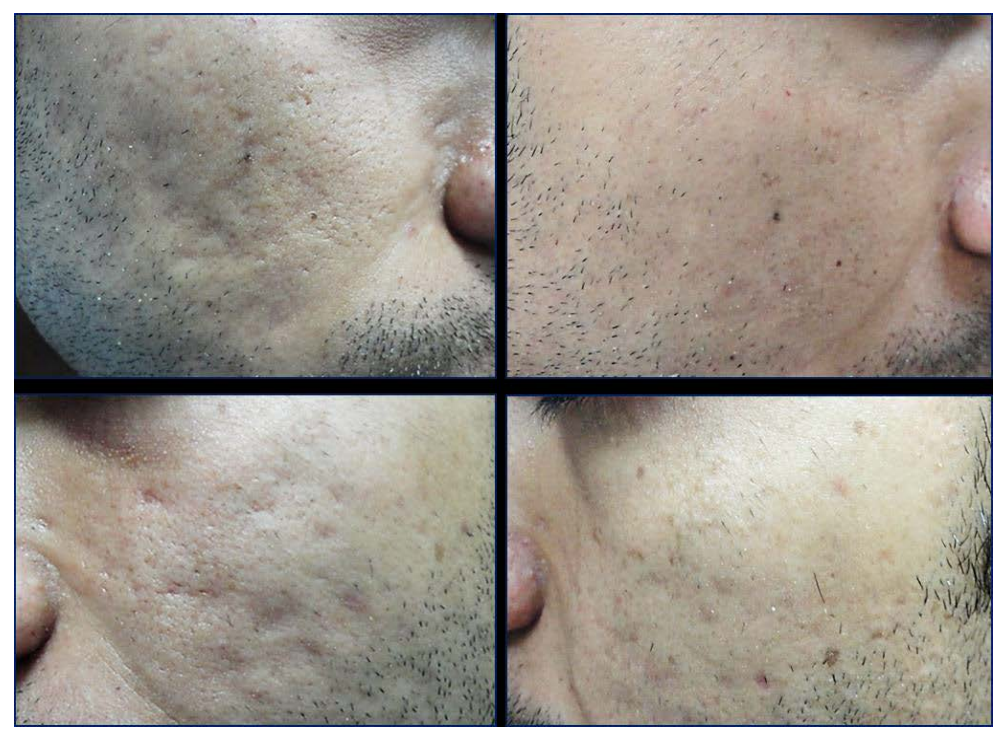

Figure 1. A patient with acne scars before and six months after subcision. 


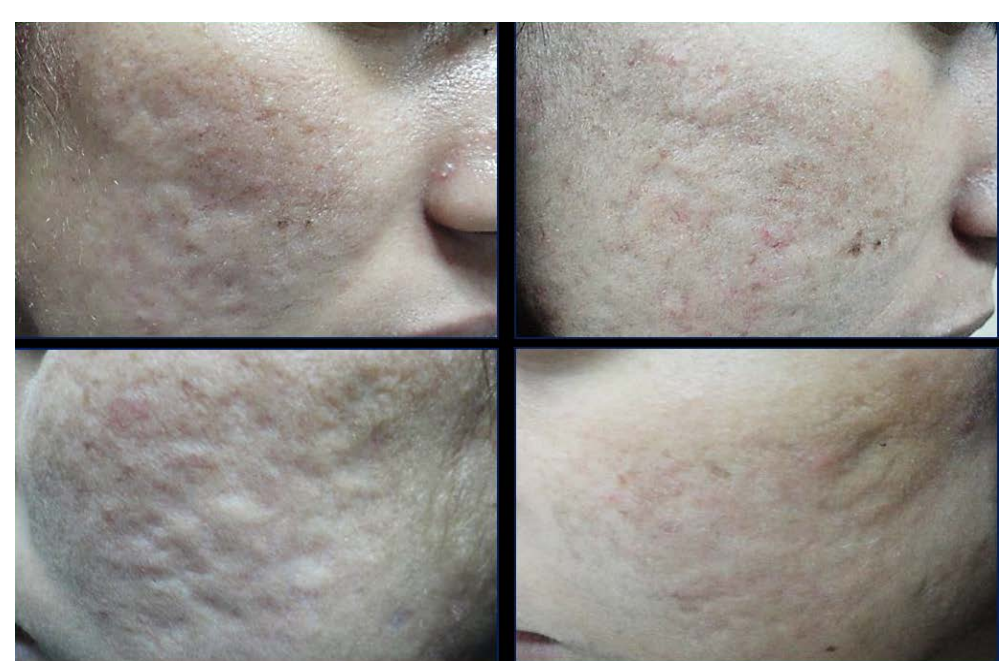

Figure 2. Patient with rolling acne scar before and six months after sub cision.

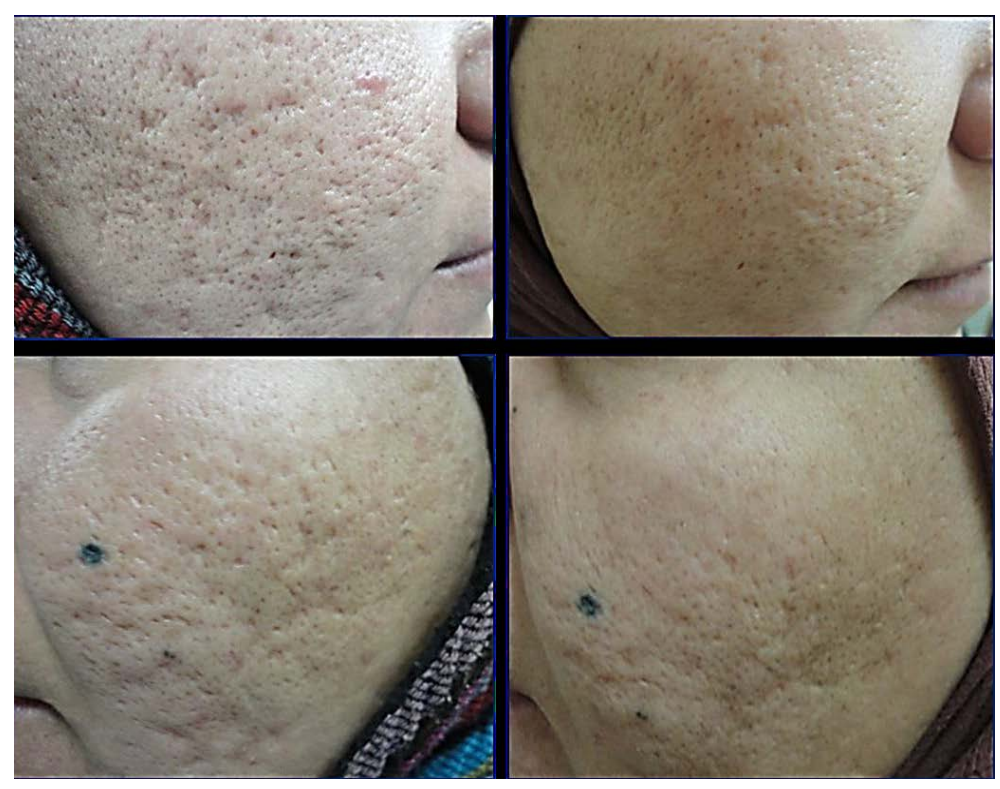

Figure 3. A patient with icepick \& boxcar acne scars before and six months after subcision.

Side Effects: All patients developed firm swelling in the treated area which disappeared gradually and at 12 weeks no patient had such a swelling. Also no patient had hyper or hypo pigmentation.

\section{Discussion}

Subcision (subcutaneous incision less surgery) is a simple procedure which is used for revision of a variety of skin depressions, including atrophic acne scars, depressed scars, wrinkles, striae, and cellulite [6]. Any area on the face can be treated with a special needle, or ordinary hypodermic needle. The term subcision is granted to David Orentreich in 1994 [7]-[9]. Subcision releases fibrous anchoring of the dermis, this together with the resultant hematoma is responsible for the immediate clinical improvement in rolling scars. After 5 to 10 days post-subcision, wrinkling of scar surface is obvious as hematoma starts resolving with the continuous healing process. Wrinkling of scar surface is a good sign, and subsequent organization of hematoma is thought to result in a new connective tissue formation. This leads to long-term correction of the defect, although this is usually not complete following the first treatment. Successive treatments appear to produce further improvement. Areas 


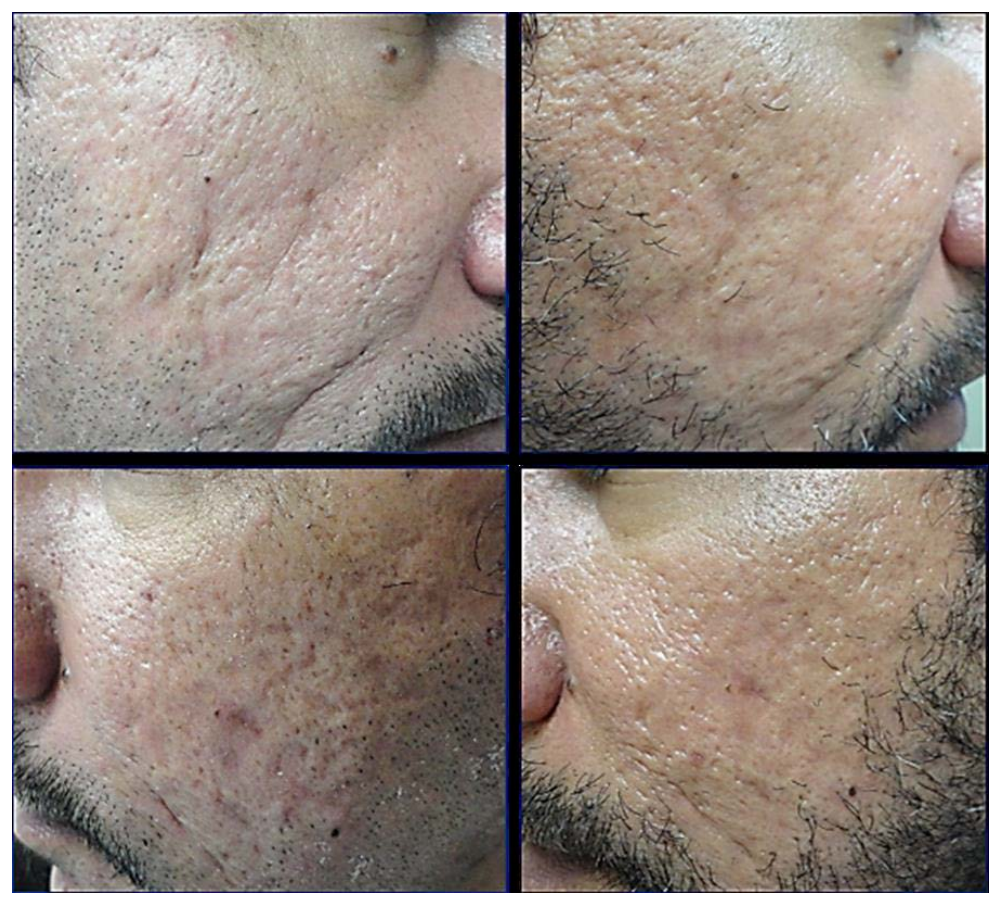

Figure 4. A patient with rolling \& icepick acne scars before and six months after subcision.

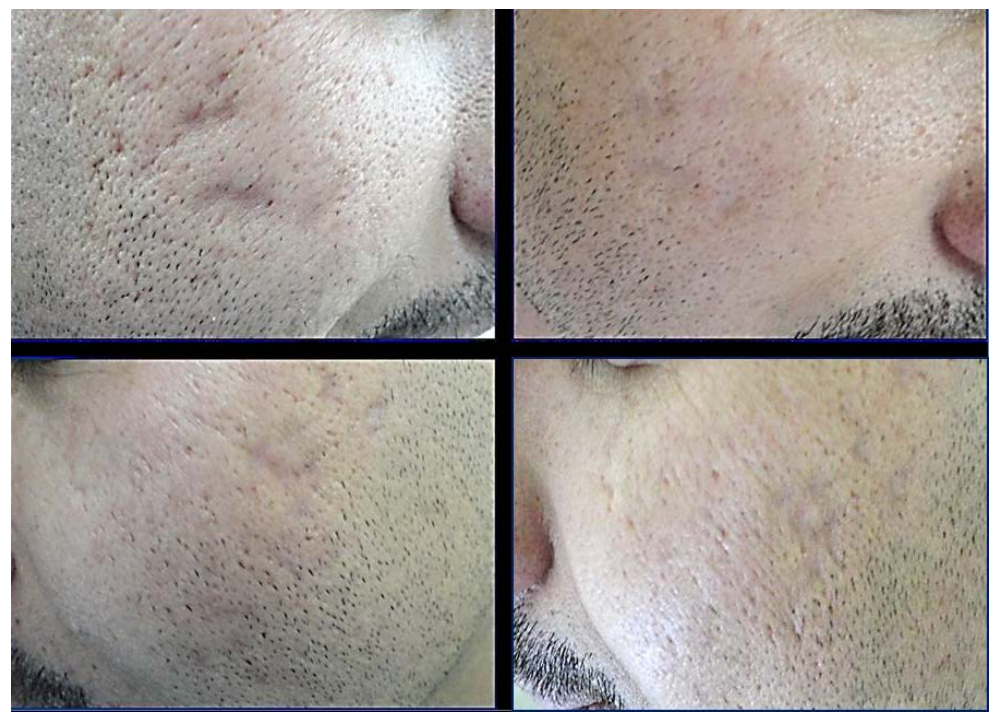

Figure 5. A patient with depressed acne scars before and six months after subcision.

of scar that appear tense should be undermined on subsequent subcision treatment. In addition to these effects subcision produces trauma at microscopic level within scar tissues. Newer matrix and collagen tissue is laid down, which is responsible for permanent clinical improvement in depressed rolling scars. Scar remodeling is a continuous process, and it cannot be considered to be in a steady state until at least 2 years post-wounding. Sur face repithelialization does not take part in the healing process since it is essentially absent. Subcision only minimally involves the epidermis.

In the present study evaluation of improvement was assessed by subjective as well as objective methods. New scoring system based on Sharquie method for grading acne scars was adopted. In addition to the total score of 
Table 1. Acne severity score, number of scars, scar area proportion of the cheek, color of scar and effect of scar on psyche in patients before treatment and at 6 months follow up.

\begin{tabular}{|c|c|c|c|c|c|c|c|c|}
\hline & & \multicolumn{2}{|c|}{ Baseline visit } & \multicolumn{2}{|c|}{$\begin{array}{l}\text { At end of } \\
\text { follow up }\end{array}$} & \multirow{2}{*}{$\boldsymbol{X}^{2}$} & \multirow{2}{*}{ df } & \multirow{2}{*}{$\mathrm{p}$ value } \\
\hline & & No. & $\%$ & No. & $\%$ & & & \\
\hline \multirow{4}{*}{$\begin{array}{c}\text { Acne scar } \\
\text { severity index }\end{array}$} & Mild score $=5-9$ & 1 & 6.2 & 8 & 50 & \multirow{4}{*}{8.13} & \multirow{4}{*}{2} & \multirow{4}{*}{0.01713441} \\
\hline & Moderate score $=10-14$ & 11 & 68.8 & 7 & 43.8 & & & \\
\hline & Severe score $=15-20$ & 4 & 25 & 1 & 6.2 & & & \\
\hline & Total & 16 & 100 & 16 & 100 & & & \\
\hline \multirow{5}{*}{ No. of scars } & $1-3$ & 3 & 18.8 & 4 & 25 & \multirow{5}{*}{5.19} & \multirow{5}{*}{3} & \multirow{5}{*}{0.15820778} \\
\hline & $4-6$ & 1 & 6.2 & 3 & 18.8 & & & \\
\hline & $7-12$ & 1 & 6.2 & 4 & 25 & & & \\
\hline & $>12$ & 11 & 68.8 & 5 & 31.2 & & & \\
\hline & Total & 16 & 100 & 16 & 100 & & & \\
\hline \multirow{5}{*}{$\begin{array}{l}\text { Scar area ratio } \\
\text { of the cheeks }\end{array}$} & $\leq 1 / 4$ & 4 & 25 & 7 & 43.8 & \multirow{5}{*}{7.42} & \multirow{5}{*}{3} & \multirow{5}{*}{0.05969840} \\
\hline & $>1 / 4-1 / 2$ & 2 & 12.5 & 6 & 37.5 & & & \\
\hline & $>1 / 2-3 / 4$ & 7 & 43.8 & 3 & 18.8 & & & \\
\hline & $>3 / 4$ & 3 & 18.8 & 0 & 0 & & & \\
\hline & Total & 16 & 100 & 16 & 100 & & & \\
\hline \multirow{3}{*}{ Type of scar } & Flat & 0 & 0 & 7 & 43.8 & \multirow{3}{*}{-} & \multirow{3}{*}{-} & \multirow{3}{*}{0.0033988} \\
\hline & Depressed & 16 & 100 & 9 & 56.2 & & & \\
\hline & Total & 16 & 100 & 16 & 100 & & & \\
\hline \multirow{4}{*}{ Color of scar } & Skin colored & 6 & 37.5 & 10 & 62.5 & \multirow{4}{*}{2.33} & \multirow{4}{*}{2} & \multirow{4}{*}{0.31140322} \\
\hline & Erythematous or hypopigmented & 2 & 12.5 & 2 & 12.5 & & & \\
\hline & Hyperpigmented & 8 & 50 & 4 & 25 & & & \\
\hline & Total & 16 & 100 & 16 & 100 & & & \\
\hline \multirow{5}{*}{ Effect on psyche } & No effect or mild discomfort & 0 & 0 & 3 & 18.8 & \multirow{5}{*}{11.85} & \multirow{5}{*}{3} & \multirow{5}{*}{0.00791507} \\
\hline & Mild bothersome & 2 & 12.5 & 8 & 50 & & & \\
\hline & Moderate bothersome & 11 & 68.8 & 5 & 31.3 & & & \\
\hline & $\begin{array}{l}\text { Severe bothersome or social } \\
\text { withdrawal }\end{array}$ & 3 & 18.8 & 0 & 0 & & & \\
\hline & Total & 16 & 100 & 16 & 100 & & & \\
\hline
\end{tabular}

${ }^{*}$ Fisher exact test.

acne severity, individual parameters such as number, area, type, and color of scar in addition to the effect of scar on the psyche were evaluated and recorded before and after treatment to reflect the improvement in the quality of scars more accurately. These details were not mentioned previously in the literatures. The total acne score decreased significantly in our patients. The scar flattened in a significant number of patients (see Figures 1-5). Half of our patients was moderately satisfied with procedure. At 6 months follow up 19\% considered their scars to have minimal effects on their lives while $50 \%$ considered them to be mildly bothersome. Regarding side effects, erythema, brusing, and swelling were the most common side effects. All disappeared at 12 week post treatment. On comparing our results to previously published studies, Alam [10] et al. evaluated the efficacy of 
subcision for the treatment of rolling acne scars in 40 patients. They demonstrated that subcision was an effective method for improving depressed scars. However evaluation depended on investigator rating and patient satisfaction. Balighi [11] et al. also used subcision for treatment of depressed acne scars in 22 patients. They concluded that subcision seemed to be a safe method to correct the rolling acne scars with long-term improvement. Vaishnani [12] stated that results after subcision differ at 2 months and 6 months, with greater improvement observed over time, because scar remodeling is a continuous process, and it cannot be considered to be in a steady state until at least 2 years after wounding [13]. Al-Dhalimi et al. demonstrated that subcision was an effective method for improving rolling acne scars in a study carried on 34 patients [8]. Subcision was compared to the application of TCA $100 \%$ individually to acne scar by Shahira et al. [14] and found subcision superior. There are a number of treatment modalities for acne scars with different degrees of success like medium depth peel [14], sand paper dermobrasion [15], and laser [15]. It is difficult to compare the results among different studies.

However in conclusion, comparing subcision to the other modalities of treatment of acne scars indicates that it is a simple procedure without much preparation. Only local anesthesia is required. It needs a maximum of 3 sessions. It is not complicated by hypo or hyper pigmentation noticed in other modalities such as chemical peel, dermobrasion and lasers [15]. Also there is no potential for scarring following subcision in contrast to dermobrasion and lasers [15]. None of our patients in the present study evaluation of improvement was assessed by subjective as well as objective methods. New scoring system based on Sharquie's method for grading acne scars was adopted. In addition to the total score of acne severity, individual parameters such as number, area, type, and color of scar in addition to the effect of scar on the psyche were evaluated and recorded before and after treatment to reflect the improvement in the quality of scars more accurately. These details were not mentioned previously in the literature. No one developed post-operative infection. Sun avoidance is not mandatory as in other surgical treatments of acne scars. All that was needed is a 2 - 3 day leave.

\section{Conclusion and Limitation}

Subcision is a good promising treatment for acne scars but we need to do it on a larger number and the patients followed up for a longer period that may yield even better results.

\section{References}

[1] Mohammad, A.F. (2008) Treatment of Acne Scars with Trichloroacetic Acid Chemical Peel Followed by Manual Dermasanding. A Thesis Submitted to the Scientific Council of Dermatology \& Venereology, Iraqi Board for Medical Specializations.

[2] Layton, A.M. (2010) Disorders of Sebaceous Glands. In: Tony, B., Stephen, B., Neil, C. and Christopher, G., Eds., Rook's Textbook of Dermatology, 8th Edition. Blackwell Scientific Publications. Oxford, UK, Chapter 42, 42.1-42.86. http://dx.doi.org/10.1002/9781444317633.ch42

[3] Cunliffe, W.J. (1986) Acne and Unemployment. British Journal of Dermatology, 115, 386. http://dx.doi.org/10.1111/j.1365-2133.1986.tb05757.x

[4] Cotteril, J.A. and Cunliffe, W.J. (1997) Suicide in Dermatological Patients. British Journal of Dermatology, 137, 246250. http://dx.doi.org/10.1046/j.1365-2133.1997.18131897.x

[5] Sharquie, K., Al-Hamdi, K., Noaimi, A.A. and Al-Battat, R. (2009) Scarring \& No Scarring Facial Acne Vulgaris \& Frequency of Associated Skin Disease. The Iraqi Postgraduate Medical Journal, 8, 332-338.

[6] Khunger, N. and Monica, K. (2011) Subcision for Depressed Facial Scars Made Easy Using a Simple Modification. Dermatologic Surgery, 37, 514-517. http://dx.doi.org/10.1111/j.1524-4725.2011.01925.X

[7] Orentreich, D.S. and Orenreich, N. (1995) Subcutaneous Incision Less (Subcision) Surgery for the Correction of Depressed Scars and Wrinkles. Dermatologic Surgery, 2, 543-549. http://dx.doi.org/10.1111/j.1524-4725.1995.tb00259.x

[8] Al-Dhalimi, M.A. and Arnoos, A.A. (2012) Subcision for Treatment of Rolling Acne Scars in Iraqi Patients: A Clinical Study. Journal of Cosmetic Dermatology, 11, 144-150. http://dx.doi.org/10.1111/j.1473-2165.2012.00616.x

[9] Fulton, J.E. and Silverton, K. (1999) Resurfacing the Acne-Scarred Face. Dermatologic Surgery, 25, 353-359. http://dx.doi.org/10.1046/j.1524-4725.1999.07229.x

[10] Alam, M., Omura, N., Omura, M. and Kaminer, M.S. (2005) Subcision for Acne Scarring: Technique and Outcome in 40 Patients. Dermatologic Surgery, 31, 310-317. http://dx.doi.org/10.1097/00042728-200503000-00011

[11] Balighi, K., Robati, R.M., Moslehi, H. and Robati, A.M. (2008) Subcision in Acne Scar with and without Subdermal Implant: Clinical Trial. Journal of the European Academy of Dermatology and Venereology, 22, 707-711. 
http://dx.doi.org/10.1111/j.1468-3083.2008.02583.x

[12] Vaishnani, J.B. (2008) Subcision in Rolling Acne Scars with 24 G Needle. Indian Journal of Dermatology, Venereology and Leprology, 74, 677-679. http://dx.doi.org/10.4103/0378-6323.45133

[13] Kim, J., Ochoa, M. and Krutzik, S. (2002) Activation of Toll-Like Receptor-2 in Acne Triggers Inflammatory Cytokine Responses. Journal of Immunology, 169, 1535-1541. http://dx.doi.org/10.4049/jimmunol.169.3.1535

[14] Shahira, A., Mohamed, H., Dalia, A. and Seham, A. (2011) Subcision Versus 100\% Trichloroacetic Acid in the Treatment of Rolling Acne Scars. Dermatologic Surgery, 37, 626-633. http://dx.doi.org/10.1111/j.1524-4725.2011.01954.x

[15] Jacob, C.I., Dover, J.S. and Kaminer, M.S. (2001) Acne Scarring: A Classification System and Review of Treatment Options. Journal of the American Academy of Dermatology, 45, 109-117. http://dx.doi.org/10.1067/mjd.2001.113451 